\title{
Locked down: Impact of COVID-19 restrictions on trauma presentations to the emergency department
}

\author{
A Venter, MB ChB; C M Lewis, FCEM (SA), MMed (Emergency Medicine), Dip PEC (SA), Dip ROM RCSEd; P Saffy, FCEM (SA), MB BCh, \\ MSc (Med), Dip PEC (SA), DA (SA); L P Chadinha, FCEM (SA), MB BCh, MMed (Emergency Medicine), BSc, Dip PEC (SA) \\ Division of Emergency Medicine, Department of Family Medicine and Primary Care, Faculty of Health Sciences, University of the Witwatersrand, \\ Johannesburg, South Africa
}

Corresponding author: A Venter(28aventer@gmail.com)

\begin{abstract}
Background. COVID-19 was recognised as a global pandemic on 11 March 2020. In South Africa (SA), a nationwide lockdown was implemented at midnight on 26 March to prepare for the predicted surge and slow the spread of the virus.

Objectives. To compare the volume and type of presentations of trauma secondary to interpersonal violence and road traffic collisions (RTCs) during two 5-month periods, from February to June 2019 and 2020, in the emergency department (ED) of an academic tertiary hospital in Gauteng Province, SA. In 2020, February - June included the lockdown period.

Methods. An observational retrospective audit of the patient register at the Helen Joseph Hospital ED was conducted, comparing the number of trauma presentations secondary to interpersonal violence (assaults with gunshot wounds, general assaults including mob assaults, assaults with stab wounds) and RTC presentations between February and June 2019 and 2020.

Results. A total of 4300 trauma presentations secondary to interpersonal violence and RTCs were noted in the 5-month period February June 2019, as opposed to 3239 presentations in February - June 2020 (25\% decline). A 40\% decline in the number of RTCs, from 1704 in February - June 2019 to 1026 in the corresponding period for 2020 , was noted and found to be statistically significant ( $p=0.03$ ). Declines in the volume of trauma cases secondary to interpersonal violence and of overall trauma cases were only directional in favour of 2020 , but not statistically significant.

Conclusions. The volume of trauma presentations secondary to interpersonal violence and RTCs in the Helen Joseph Hospital ED decreased during the lockdown period. The decline in the volume of RTCs was statistically significant, but declines in the volume of trauma presentations secondary to interpersonal violence and in the volume of overall trauma presentations were not.
\end{abstract}

S Afr Med J 2021;111(1):52-56. https://doi.org/10.7196/SAMJ.2021.v111i1.15289

COVID-19 was recognised as a global pandemic on 11 March 2020. It has been spreading fast across the world since the initial outbreak in December 2019 in Wuhan, China, and many countries have experienced a level of self-isolation and travel restrictions. ${ }^{[1]}$ On 5 March 2020, the first positive case of COVID-19 was diagnosed in South Africa (SA), and President Cyril Ramaphosa announced a national lockdown that started at midnight on 26 March. $^{[2,3]}$

Lockdown, in the context of COVID-19, can be referred to as public health and social measure actions taken by governments, communities, institutions and individuals to slow or stop the spread of the virus. In SA, these measures included social and physical distancing; restricting international, interprovincial and non-essential travel; prohibiting mass gatherings (except for funeral gatherings of up to 50 people); closure of non-essential facilities and services; and a ban on the sale of cigarettes and alcohol. ${ }^{[4]}$ Lockdown in SA is divided into five levels, determined by a risk-adjusted strategy, with level 5 enforcing drastic measures to contain the spread of COVID-19 and subsequent levels easing restrictions and allowing more movement and greater economic activity. Level 5, initiated on 26 March, was stepped down to level 4 on 1 May, with further step-downs to level 3 on 1 June, level 2 on 18 August and level 1 on 21 September, which remained in force at the time of writing. ${ }^{[5]}$

Although lockdown has devastating social, economic and even psychological implications, ${ }^{[1]}$ it is possible that it could have secondary effects such as a decrease in interpersonal violence and road traffic collisions (RTCs) across the country.

\section{Objectives}

The lockdown period provided an opportunity to study the effect that the restrictive measures implemented have had in an urban setting in Gauteng Province, SA. This study aimed to provide a quantitative assessment of the volume of trauma cases secondary to interpersonal violence and RTCs, comparing the same 5-month periods in 2019 and 2020, the latter inclusive of the lockdown period, in this setting.

\section{Methods}

An observational retrospective audit of the patient register in the Helen Joseph Hospital Emergency Department (ED) was conducted to describe and compare the volume and type of presentations of interpersonal violence and RTCs in two 5-month periods from February to June 2019 and 2020 in an academic tertiary hospital in Gauteng. This time frame encompasses the time during which SA developed its initial response to the COVID-19 pandemic, inclusive of a nationwide lockdown with all South Africans, other than those providing essential services, confined to their homes unless grocery shopping, attending a pharmacy or seeking medical attention.

This retrospective study included all trauma presentations secondary to interpersonal violence and RTCs in the ED of Helen Joseph Hospital, Johannesburg, from 1 February 2019 to 30 June 2019 and 1 February 2020 to 30 June 2020. This public hospital provides tertiary healthcare to a population of $\sim 1$ million low- to middleincome people. The ED attends to $\sim 60000$ patients annually. The vast majority of patients seen are adults, as paediatric patients attend 
a mother and child hospital nearby. Areas that Helen Joseph Hospital serve include regions $\mathrm{B}$ and $\mathrm{C}$ and parts of region $\mathrm{A}$ of the Johannesburg metropolitan area. ${ }^{[6}$

All patients are manually entered into a register book by the ED staff on presentation. This includes the presenting complaint and mechanism of injury according to the patient, a friend or family member, or police or emergency medical services. Data variables collected for our study were extracted manually from the register book by counting the number of assaults with gunshot wounds, general assaults including mob assaults, assaults with stab wounds (all considered to be trauma secondary to interpersonal violence) and RTCs, including motor vehicle collisions, pedestrian-vehicle collisions and motorbike collisions, each month under these categories. When a single patient sustained multiple modes of trauma, for example mob assault and stab wound, the primary mode was documented by the triage personnel at their discretion. The register book was the only data source, and data were captured anonymously.

Permission to use data from the register book was obtained from the ethics committee at Helen Joseph Hospital, and ethics approval was granted by the Human Research Ethics Committee of the University of the Witwatersrand (ref. no. M200738).

Using a password-protected laptop, data were captured in Excel, version 365 (Microsoft, USA), on a spreadsheet. The data were analysed using SPSS Statistics, version 22 (IBM, USA). The data were numerical and continuous, in the form of monthly counts of trauma cases, and the descriptive statistics were therefore presented as means, standard deviations, medians and interquartile ranges. Tests of normality showed that the data deviated significantly from a normal distribution (i.e. $p<0.05$ ). Non-parametric (distributionfree) statistical methods were therefore used in the subsequent analysis. The Mann-Whitney $U$-test was used to compare differences in the number of trauma cases for the period February - June 2019 compared with February-June 2020. Significance testing was set at the $95 \%$ confidence level.

\section{Results}

From February to June 2019, a total of 4300 trauma presentations secondary to either interpersonal violence or RTCs were seen in the ED. From February to June 2020, the total number of trauma presentations with the same causes decreased to 3239 . This represents an overall reduction of $25 \%$ for the 5-month period.

Four major categories, including assaults with gunshot wounds, general assaults including mob assaults, assaults with stab wounds (all considered to be trauma secondary to interpersonal violence) and RTCs, including motor vehicle collisions, pedestrian-vehicle collisions and motorbike collisions, were used for the purposes of analysis. The volume of trauma presentations per category over the 5-month period for 2019 against 2020 is shown in Fig. 1.

There were declines in the number of trauma cases for all the trauma categories, as well as overall, in February - June 2020 compared with February - June 2019 (Fig. 2).

There were 489 fewer trauma presentations secondary to interpersonal violence and RTCs in April 2020 and 502 fewer in May 2020 compared with the same months in the preceding year, representing $62 \%$ and $55 \%$ declines, respectively, corresponding to when lockdown regulations were the most limiting/strict (levels 5 and 4). Compared

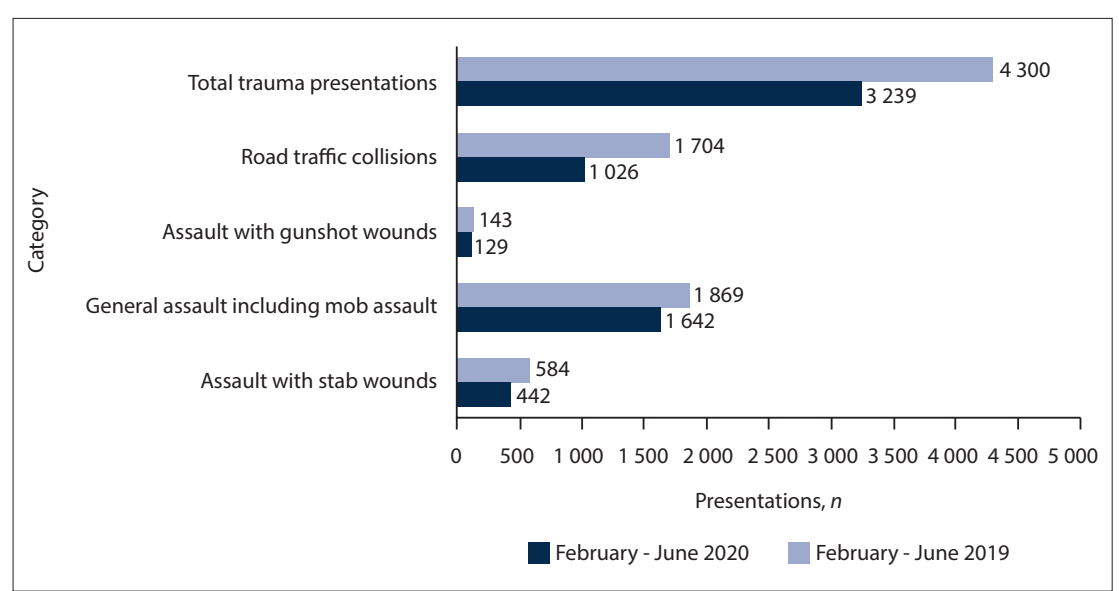

Fig. 1. Volume of trauma presentations, February - June 2019 and 2020.

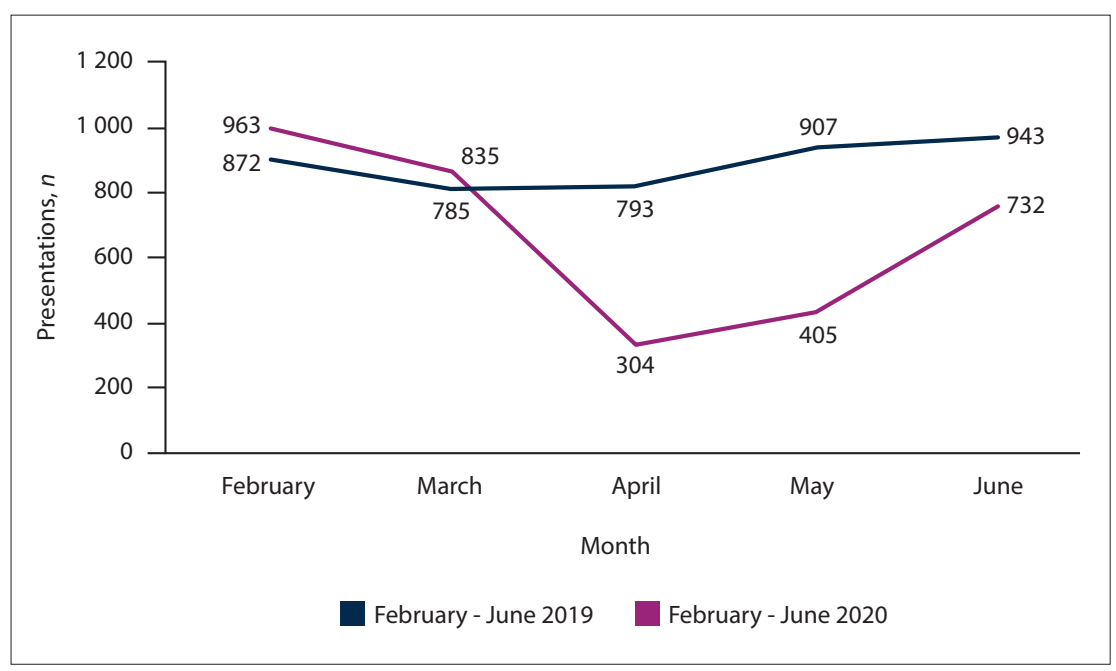

Fig. 2. Trend of trauma presentations, 2019 and 2020. with June 2019, there were 211 fewer cases in June 2020 (22\% decline), when lockdown restrictions had been partially lifted/eased.

\section{Changes per category for each month of lockdown in 2020 compared with 2019}

Assaults with gunshot wounds declined by 8 cases (28\% decline) in April 2020, by 16 cases (55\% decline) in May 2020 and by with the same months in 2019.

General assaults, including mob assaults, declined by 205 cases ( $57 \%$ decline) in April 2020 , by 137 cases (38\% decline) in May 2020 and by 50 cases (11\% decline) in June 2020 compared with the same months in 2019

Assaults with stab wounds declined by 51 cases (46\% decline) in April 2020, by 60 cases (58\% decline) in May 2020 and by 35 cases (33\% decline) in June 2020 compared with the same months in 2019.

RTCs declined by 225 cases ( $77 \%$ decline) in April 2020, by 289 cases (71\% decline) in 6 cases (19\% decline) in June 2020 compared 
May 2020 and by 121 cases (35\% decline) in June 2020 compared with the same months in 2019.

\section{Percentage change in trauma cases,}

\section{February - June 2019v. February - June 2020}

There were declines in the number of trauma cases for all trauma categories, as well as overall, in February - June 2020 compared with February - June 2019 (Table 1).

The 40\% decline in the number of RTCs from 1704 in February June 2019 to 1026 in the corresponding period for 2020 was statistically significant $(p=0.03)$ when the Mann-Whitney $U$-test was applied. However, the declines in the number of other categories of trauma cases were only directional in favour of 2020, but not statistically significant.

\section{Discussion}

SA has a high burden of interpersonal violence and RTCs, which impose a heavy economic and health burden on the country. It has been postulated that this high burden may be a result of the misuse of alcohol, among other contributory factors. ${ }^{[7,8]}$

This study describes the volume of trauma seen during a 5-month period, comparing 2019 with 2020 . There was no significant difference in month-to-month variation in 2019. However, comparing April, May and June 2020 with the same months in 2019, the decrease in the volume of trauma seen (probably due to the effect of the lockdown) is noteworthy, if not statistically significant.

The nationwide lockdown that was implemented at midnight on 26 March 2020 is thought to have had a significant impact on the number of trauma presentations to the $\mathrm{ED}$, with a total reduction of 489 cases (62\% decline) in April, 502 cases (55\% decline) in May and 211 cases (22\% decline) in June compared with 2019. Similar lockdown measures implemented elsewhere have led to safety improvement and a reduction in crime and trauma presentations in various cities across the globe. ${ }^{[1]}$

Morris et al ${ }^{[9]}$ found a $47 \%$ reduction in trauma cases for April 2020 compared with April 2018 and 2019 in a recent study in the Edendale Hospital ED in KwaZulu-Natal (KZN) Province, SA. Their study showed a major decrease in motor vehicle collisions, pedestrian-vehicle collisions, assaults and gunshot wounds.

In our study, the most significant reductions in ED presentations were seen in the category of RTCs, with 225 fewer cases (78\% decline) in April 2020, 289 fewer cases (71\% decline) in May and 121 fewer cases (35\% decline) in June compared with 2019.

It is likely that the cause of the reduction in trauma noted in our study is multifactorial, including, but not limited to, restriction of access to alcohol, a decrease in the number of vehicles on the road, less highrisk behaviour when driving (such as speeding, alcohol consumption, overloading of vehicles and failure to wear seatbelts ${ }^{[10]}$ ), an enforced curfew, restrictions on public gatherings, and increased visible policing.

One of the measures taken during lockdown in SA was restriction of access to alcohol, which has been suggested as one of the factors contributing to the decreased incidence of assaults secondary to interpersonal violence and road accidents. ${ }^{[11]}$

Parry et al. ${ }^{[8]}$ noted that in 2015, 62300 adults died (95\% uncertainty interval 27000 - 103000 ) in SA from alcohol-attributable causes. In SA, alcohol is the fifth largest risk factor for death and disability. South Africans consume $\sim 50 \%$ more alcohol than the World Health Organization African Region in total, and levels of heavy episodic drinking are extremely high. Research studies conducted over a 50-year period and published by the South African Medical Research Council (SAMRC) reported a high burden of harm associated with alcohol use, and there is growing recognition of the need for national action to reduce this harm. ${ }^{[8]}$ According to Sun et al., ${ }^{[12]}$ alcohol is a major contributor to disease, domestic violence, injuries and death. Restricting access to alcohol results in a reduction of alcohol-related mortality, crime and injuries, according to documentation in a number of substantial articles in the economics literature. ${ }^{[12]}$

Pawar et al. ${ }^{[13]}$ assessed the impact of physical distancing measures on daily travel for work during India's initial response to the COVID19 pandemic, and noted a $41 \%$ reduction in commuters during the transition to India's COVID-19 lockdown response. Thornton ${ }^{[14]}$ noted a 25\% decrease in visits to EDs in England during the week following commencement of lockdown in the UK on 23 March 2020. It was suggested that there may be less disease due to social distancing and fewer RTCs secondary to decreased motor vehicle use. It is reasonable to infer that restrictions placed on individual mobility and workplace activity would have resulted in a decreased number of vehicles on the road during SA's lockdown response, with the number of vehicles on the road increasing steadily with easing of restrictions. The decrease in number of vehicles on the road may have contributed to the decrease in RTCs noted in our study, which similarly increased as restrictions were eased.

Parkinson et al.$^{[15]}$ previously described the contribution of highrisk behaviours, including speeding, alcohol intoxication, failure to wear seatbelts and overloading of vehicles, to the high burden of RTCs in the SA context. Our study did not specifically investigate the presence of high-risk behaviours in trauma patients presenting to the ED, and a more detailed study would be needed to determine whether these factors were contributory to RTC presentations.

Reductions in interpersonal violence, including assaults with gunshot wounds, general assaults including mob assaults and assaults with stab wounds, also require further investigation. 'Street violence' (such as the assaults described above) has been noted to have decreased in many parts of the world, including SA, Latin America and parts of the USA, during their respective lockdown periods, and this is thought to be a result of closure of bars and nightclubs (inclusive of restricted access to alcohol) and curfew restrictions. ${ }^{[10]}$ Saimen et al. ${ }^{[16]}$ reported that interpersonal violence was ranked fourth as a leading single cause of all disability-adjusted life-years in SA in 2017, and alcohol use has been identified as the fourth leading risk factor driving the most death and disability combined. ${ }^{[17]}$ It is

Table 1. Trauma presentations, February - June 2019v. February - June 2020

\begin{tabular}{|c|c|c|c|c|}
\hline Trauma category & Feb - Jun 2019, $n$ & Feb - Jun 2020, $n$ & Change, $\%$ & $p$-value \\
\hline Assault with gunshot wounds & 143 & 129 & -10 & 0.60 \\
\hline General assault including mob assault & 1869 & 1642 & -12 & 0.60 \\
\hline Assault with stab wounds & 584 & 442 & -32 & 0.25 \\
\hline Road traffic collisions & 1704 & 1026 & -40 & $0.03^{*}$ \\
\hline Total trauma presentations & 4300 & 3239 & -25 & 0.25 \\
\hline
\end{tabular}


possible that restricted access to alcohol and an enforced curfew may be factors contributing to the decreased burden of interpersonal violence-related trauma presentations noted in our study over the SA lockdown period.

It is difficult to quantify what role, if any, primary prevention strategies such as the visible policing during the SA lockdown response had on the reduction in interpersonal violence and RTCs apparent in our study.

It was postulated by Thornton, ${ }^{[14]}$ when considering causative factors for a $25 \%$ reduction in visits to EDs in the week following lockdown implementation in England, that people may have chosen to wait before attending the ED when sickness occurs because they were worried about contracting COVID-19 at the ED, thus ultimately resulting in fewer ED presentations. Morris et al. ${ }^{[9]}$ in their recent study in KZN assessing trauma presentations in April 2020 compared with April 2018 and 2019, considered that this was probably not a causative factor. They supported this hypothesis by demonstrating that, although the number of trauma presentations decreased by $47 \%$, the severity of presentations in their cohort in 2020 did not differ significantly from that in previous years. If a reduction in trauma presentations was due to a reduction in health-seeking behaviour secondary to fear, it would be expected that the proportion of mild to moderate injury severity presentations would decrease in comparison with more severe injuries, which was not noted. ${ }^{[9]}$

According to the Rapid Mortality Reporting System (developed by the Burden of Disease Unit, SAMRC, and the University of Cape Town's Centre for Actuarial Research), actual deaths over the first 5 weeks of lockdown decreased from an expected 800 deaths per week (with an anticipated peak of 1000 deaths per week over the Easter period) to 400 deaths per week. ${ }^{[1]]}$ This contrasts with many countries that experienced an excess of deaths due to COVID-19 at a similar stage of their response to the COVID-19 pandemic. Unnatural deaths in SA, many of which are attributed to interpersonal violence and RTCs, halved during the initial restrictive stage of lockdown. ${ }^{[11]}$

As several measures likely to have contributed to the decrease in interpersonal violence and RTCs noted in our study were implemented at the same time, we are unable to ascertain which of these measures had the most significant effect. On 12 July 2020, the sale and distribution of alcohol ban was reimplemented in SA after initially being lifted on 1 June, and the curfew regulations were amended to impose a curfew from $22 \mathrm{~h} 00$ to $04 \mathrm{~h} 00$ daily (as opposed to the preceding curfew from $21 \mathrm{~h} 00$ to $04 \mathrm{~h} 00$ ), while other restrictions remained eased. These different combinations of circumstances may allow future investigators an opportunity to investigate the impact that alcohol, and to a lesser extent curfew, restrictions had on trauma presentations more clearly.

While our study has highlighted a decrease in interpersonal violence and RTC presentations, we have not assessed the potential negative effects of lockdown as they pertain to healthcare. Studies have noted increases in suicide, gender-based violence and domestic abuse, which are thought to be secondary to increased social stressors brought about by a combination of the COVID-19 pandemic and lockdown measures implemented in many countries in response to the pandemic. ${ }^{[10]}$ We have not assessed the cost of a decrease in healthseeking behaviour to patients with chronic medical conditions, or the economic impact of lockdown measures on healthcare systems.

\section{Study limitations}

This study is limited by the accuracy of the patient register books in the Helen Joseph Hospital ED. Presenting complaint and mechanism of injury for each patient are manually recorded, and the study is therefore limited by the personnel's discretion and accuracy of data entered.

\section{Conclusions}

Interpersonal violence- and RTC-related trauma presentations to the Helen Joseph Hospital ED decreased during the period of lockdown implemented in response to the COVID-19 pandemic. It is noteworthy, however, that although the decrease in RTCs was statistically significant, the decrease in interpersonal violence and overall trauma observed in our study was not, suggesting that lockdown measures failed to significantly impact on the burden of trauma seen in the ED. As lockdown measures were eased, a rise in the number of ED trauma presentations was observed. While it is impossible to ascertain which of the lockdown measures taken contributed most significantly to the reduction in trauma presentations noted, we can conclude that together these restrictions did result in a reduction in the burden of trauma seen in the ED, albeit without statistical significance. These results could be compared with other hospitals across the country and could also be compared with studies undertaken that encompass the time frame from July 2020 onwards, as this may provide more clarity on the role that alcohol, and to a lesser extent curfew, restrictions have on the incidence of trauma presentations. These studies could then be used to guide public health interventions aimed at mitigating the high burden of interpersonal violence and RTCs in the SA setting.

\section{Declaration. None}

Acknowledgements. Successful conduct of this study was made possible by the Helen Joseph Hospital ED staff.

Dissemination of results. Results from this study will be disseminated through publication as well as shared with staff members at the data collection site through an informal presentation.

Author contributions. All authors conceived the study idea, CML and PS designed the data collection tool, all authors collected the data, LPC analysed the data, and AV drafted the manuscript. All authors revised and approved the final manuscript.

Funding. None.

Conflicts of interest. None.

1. Shayegh S, Malpede M. Staying home saves lives, really! SSRN, 3 April 2020. https://doi.org/10.2139/ ssrn. 3567394

2. National Institute for Communicable Diseases. First case of COVID-19 coronavirus reported in SA. 5 March 2020. https://www.nicd.ac.za/first-case-of-covid-19-coronavirus-reported-in-sa/ (accessed 9 June 2020).

3. Hamzelou J. World in lockdown. New Scientist 2020;245(3275):7. https://doi.org/10.1016/S02624079(20)30611-4

World Health Organization. Considerations in adjusting public health and social measures in the context of COVID-19: Interim guidance. 16 April 2020. https://apps.who.int/iris/handle/10665/331773 (accessed 10 June 2020).

5. South African Government. COVID-19/novel coronavirus. 2020. https://www.gov.za/Coronavirus (accessed 1 September 2020).

6. Gauteng Province - Health. Helen Joseph Hospital - Auckland Park, Johannesburg, Gauteng, South Africa. http://doctors-hospitals-medical-cape-town-south-africa.blaauwberg.net/hospitals_ South Africa. http://doctors-hospitals-medical-cape-town-south-africa.blaauwberg.net/hospitals_
clinics_state_hospitals/state_public_hospitals_clinics_gauteng_south_africa/helen_joseph_hospital_ clinics_state_hospitals/state_public_hospitals_clinics_gauteng_south_africa//
auckland_park_johannesburg_gauteng_south_africa (accessed 9 June 2020).

7. Matzopoulos R, Abrahams N, Bowman B, et al. Utility of crime surveys for Sustainable Development . Matzopoulos R, Abrahams N, Bowman B, et al. Utility of crime surveys for Sustainable Development
Goals monitoring and violence prevention using a public health approach. S Afr Med J 2019;109(6):382Goals monitoring and violence prevention using a public

8. Parry C, Myers B, Matzopoulos R, et al. Looking back, moving forward: 50 years of South African Medical Research Council alcohol-related publications. S Afr Med J 2019;109(11b):30-35. https://doi. org/10.7196/SAMJ.2019.v109i1 1b.14277

9. Morris D, Rogers M, Kissmer N, et al. Impact of lockdown measures implemented during the Covid-19 pandemic on the burden of trauma presentations to a regional emergency department in Kwa-Zulu Natal, South Africa. Afr J Emerg Med 2020 (epub 16 June 2020). https://doi.org/10.1016/j. afjem.2020.06.005

10. Papadimos TJ. What's new in critical illness and injury science? Mental health and COVID-19: Selfinflicted and interpersonal violence amid a pandemic. Int J Crit Illn Inj Sci 2020;10(2):45-48. https:// doi.org/10.4103/IJCIIS.IJCIIS_66_20 
11. MOVENDI International. Alcohol sales ban in South Africa: Benefits and big alcohol opposition. 13 May 2020. https://movendi.ngo/news/2020/05/13/alcohol-sales-ban-in-south-africa-benefits-andbig-alcohol-opposition/ (accessed 4 June 2020).

12. Sun W, Jian L, Xiao J, Akesson G, Somerford P. The impact of alcohol restriction on hospital and emergency department service utilizations in two remote towns in the Kimberley Region of Western Australia. Front Public Health 2019;7:17. https://doi.org/10.3389/fpubh.2019.00017

13. Pawar D, Yadav A, Akolekar N, Velaga N. Impact of physical distancing due to novel coronavirus (SARS-CoV-2) on daily travel for work during transition to lockdown. TRIP 2020;7:100203. https:/L doi.org/10.1016/j.trip.2020.100203

14. Thornton J. Covid-19: A\&E visits in England fall by $25 \%$ in week after lockdown. BMJ 2020;369:m1401. https://doi.org/10.1136/bmj.m1401
15. Parkinson F, Kent A, Aldous C, Oosthuizen G, Clarke D. Road traffic crashes in South Africa: The burden of injury to a regional trauma centre. S Afr Med J 2013;103(11):850-852. https://doi.

16. Saimen A, Gordon G, Govender I. Non-fatal injuries of interpersonal violence at the Leratong Provincial Hospital, South Africa. S Afr Fam Pract 2016;58(3):80-86. https://doi.org/10.1080/20786190.2016.1167311 17. Healthdata.org. South Africa: Institute for Health Metrics and Evaluation. C2017. http://www. healthdata.org/south-africa (accessed 9 June 2020)

Accepted 12 November 2020. 